\title{
Ear drops containing steroids were better than acetic acid for otitis externa
}

van Balen FA, Smit WM, Zuithoff NP, et al. Clinical efficacy of three common treatments in acute otitis externa in primary care: randomised controlled trial. BMJ 2003;327:1201-5.

\section{$Q$ In patients with acute otitis externa, what is the relative effectiveness of ear drops containing acetic acid, steroids and acetic acid, and steroids and antibiotics?}

\section{METHODS}

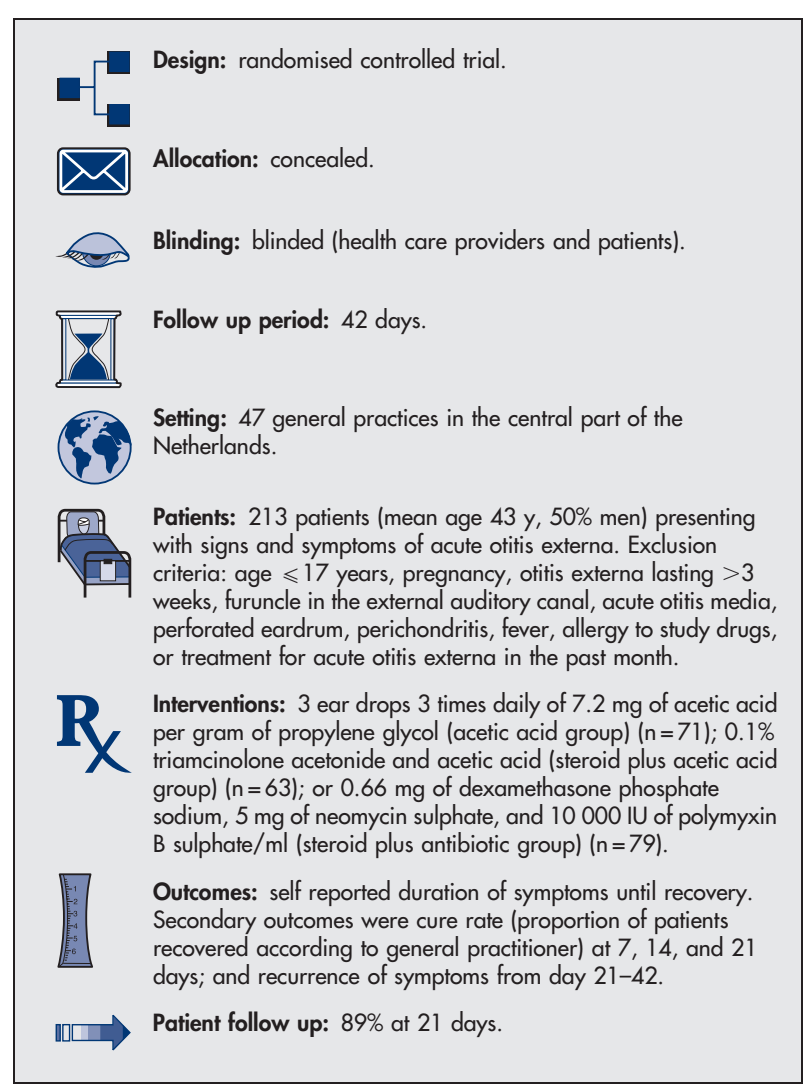

\section{MAIN RESULTS}

Analysis was by intention to treat. Duration of symptoms was shorter in patients who received steroids plus acetic acid or steroids plus antibiotics than in those who received acetic acid (mean $7 v 8 \mathrm{~d}$ and 6

For correspondence: Dr F A van Balen, Julius Center for Health Sciences and Primary Care, Utrecht, Netherlands. f.a.m.vanbalen@med.uu.nl

Sources of funding: Dutch College of General Practitioners and Foundation for the Advancement of Appropriate Drug Usage. $v 8 \mathrm{~d}$, respectively, $\mathrm{p}<0.001$ across 3 groups). The cure rate was greater at 14 and 21 days in patients who were in the steroid plus acetic acid or steroid plus antibiotic groups than those in the acetic acid group (table). Fewer patients in the steroid plus acetic acid group than the acetic acid group had a recurrence of otitis externa at 21-42 days (table).

\section{CONCLUSION}

Ear drops containing steroids with acetic acid or antibiotics were more effective than acetic acid alone for acute otitis externa.

\section{Commentary}

cute otitis externa, an infection of the external auditory canal, is
associated with exposure to warm humid climates, swimming, and
aggressive cleaning of the ear canal. ${ }^{1}$ It is a common disease in
primary care, usually treated with ear drops containing antibiotics with
steroids; however, the optimal treatment has not been established. The
study by van Balen et al is the first large study of acute otitis externa that
examined cure rates at 7 days and recurrence after symptom resolution.
Based on the findings, the authors recommend that acetic acid drops
alone should no longer be used to treat adults with acute otitis externa.
Methodological strengths of this study include recruitment of patients
from 47 general practices, concealed allocation of patients, blinding of
patients and clinicians, training of general practitioners in diagnosing
and treating acute otitis externa, and inclusion of important clinical
endpoints, such as speed of recovery and symptom recurrence up to 42
days after treatment initiation. Adjustment of the primary and secondary
outcomes for differences in baseline characteristics improved the rigour
of the study. Confidence intervals around odds ratios reported in the
study and around the relative benefit increases and the relative risk
reductions reported in the table of the EBN abstract indicate a lack of
precision that would have been improved with a larger sample size.
The study makes a strong case for the use of steroids combined with
either acetic acid or antibiotics for treatment of acute otitis externa. Cau-
tion should be exercised when applying these findings to patients with
comorbid conditions because this study predominantly included healthy
adults. The results are particularly relevant to nurse practitioners working in
primary healthcare and ear-nose-throat clinical settings.
Sarah Davies RN(EC), BScN, PHCNP
Hamilton Community Health Centre-PCN
Angela de Guzman RN(EC), BScN, PHCNP
1 Uphold CR, Graham MV. Clinical guidelines in family practice. Fourth
edition. Gainesville: Barmarrae Books, 2003 .
McMaster University, Hamilton, Ontario, Canada
Masters in Clinical Health Science (Nurin) Student

Steroids plus acetic acid $(S+A A)$ or steroids plus antibiotics $(S+A B) v$ acetic acid $(A A)$ for acute otitis externa*

\begin{tabular}{|c|c|c|c|c|}
\hline Outcomes & Comparisons & Event rates & RBI $(95 \%$ Cl) & NNT (Cl) \\
\hline \multirow{2}{*}{ Cure rate at 14 days } & $\mathrm{S}+\mathrm{AA} \vee \mathrm{AA}$ & $75 \%$ v $57 \%$ & $33 \%(4$ to 54$)$ & $6(4$ to 44$)$ \\
\hline & $S+A B \vee A A$ & $82 \%$ v $57 \%$ & $44 \%$ (19 to 60$)$ & $4(3$ to 10$)$ \\
\hline \multirow{3}{*}{ Cure rate at 21 days } & $S+A A \vee A A$ & $89 \%$ > $62 \%$ & $45 \%$ (23 to 54 ) & $4(3$ to 7$)$ \\
\hline & $S+A B \vee A A$ & $86 \%$ v $62 \%$ & $39 \%$ (19 to 51$)$ & $5(4$ to 9$)$ \\
\hline & & & RRR (CI) & NNT (Cl) \\
\hline \multirow[t]{2}{*}{ Recurrence at $21-42$ days $\dagger$} & $\mathrm{S}+\mathrm{AA} \vee \mathrm{AA}$ & $26 \% \vee 45 \%$ & $56 \%$ (19 to 83 ) & 4 (3 to 12$)$ \\
\hline & $S+A B \vee A A$ & $21 \% \vee 45 \%$ & $45 \%$ (0 to 69$)$ & Not significant \\
\hline
\end{tabular}

*Abbreviations defined in glossary; RBI, RRR, NNT, and Cl calculated from data in article using odds ratios adjusted for differences at baseline. †Based on 172 patients who were symptom free at day 21. 